\title{
Thrombospondin-1 triggers macrophage IL-10 production and promotes resolution of experimental lung injury
}

\author{
Y Zhao $^{1}$, Z Xiong ${ }^{1}$, EJ Lechner ${ }^{1}$, PA Klenotic ${ }^{2}$, BJ Hamburg ${ }^{1}$, M Hulver ${ }^{1}$, A Khare ${ }^{1}$, T Oriss ${ }^{1}$, \\ N Mangalmurti ${ }^{3}, \mathrm{Y} \mathrm{Chan}^{1}$, Y Zhang ${ }^{1}$, MA Ross ${ }^{4}$, DB Stolz ${ }^{4}$, MR Rosengart ${ }^{5}$, J Pilewski ${ }^{1}$, P Ray $^{1}$, A Ray $^{1}$, \\ RL Silverstein ${ }^{6}$ and JS Lee ${ }^{1,7}$
}

Mononuclear phagocyte recognition of apoptotic cells triggering suppressive cytokine signaling is a key event in inflammation resolution from injury. Mice deficient in thrombospondin (TSP)-1 (thbs $1^{-/}$), an extracellular matrix glycoprotein that bridges cell-cell interactions, are prone to lipopolysaccharide-induced lung injury and show defective macrophage interleukin (IL)-10 production during the resolution phase of inflammation. Reconstitution of IL-10 rescues thbs $1^{-I^{-}}$mice from persistent neutrophilic lung inflammation and injury and thbs $1^{-1}$ alveolar macrophages show defective IL-10 production following intratracheal instillation of apoptotic neutrophils despite intact efferocytosis. Following co-culture with apoptotic neutrophils, thbs $1^{-I^{-}}$macrophages show a selective defect in IL-10 production, whereas prostaglandin E2 and transforming growth factor beta 1 responses remain intact. Full macrophage IL-10 responses require the engagement of TSP-1 structural repeat 2 domain and the macrophage scavenger receptor CD36 LIMP-II Emp sequence homology (CLESH) domain in vitro. Although TSP-1 is not essential for macrophage engulfment of apoptotic neutrophils in vivo, TSP-1 aids in the curtailment of inflammatory responses during the resolution phase of injury in the lungs by providing a means by which apoptotic cells are recognized and trigger optimal IL-10 production by macrophages.

\section{INTRODUCTION}

Acute respiratory distress syndrome (ARDS) is diffuse lung injury, the hallmark of which is increased pulmonary vascular permeability, and characterized pathologically by widespread alveolar damage with hyaline membrane formation, edema, hemorrhage, and inflammation. ${ }^{1}$ Although the precise etiology of ARDS is not understood, what is known is that divergent clinical risk factors such as sepsis, multiple trauma, aspiration, pneumonia, pancreatitis, and multiple transfusions pre-dispose individuals toward the development of lung injury. ${ }^{1}$ One enigmatic feature of ARDS is that only a minority of individuals with a known risk factor actually progress to lung injury ${ }^{2}$ suggesting host mechanisms that protect or promote resolution following injurious stimuli are likely involved in determining the susceptibility of a host.

Neutrophilic inflammation is a distinctive feature of human ARDS. ${ }^{3}$ Following the initial encounter with and response to damage, neutrophils senesce and can undergo apoptosis. Clearance of apoptotic neutrophils expressing surface phosphatidylserine promotes the successful curtailment of inflammation and resolution of injury. This restoration to homeostasis requires the coordinated recognition and phagocytosis of apoptotic cells by macrophages and, in experimental models, the production of anti-inflammatory cytokines

\footnotetext{
${ }^{1}$ Division of Pulmonary, Allergy, and Critical Care Medicine, Department of Medicine, University of Pittsburgh, Pittsburgh, Pennsylvania, USA. ${ }^{2}$ Department of Cell Biology, Lerner Research Institute, Cleveland Clinic, Cleveland, Ohio, USA. ${ }^{3}$ Division of Pulmonary, Allergy, and Critical Care, Department of Medicine, University of Pennsylvania, Philadelphia, Pennsylvania, USA. ${ }^{4}$ Department of Cell Biology and Center for Biological Imaging, University of Pittsburgh, Pittsburgh, Pennsylvania, USA. ${ }^{5}$ Division of Trauma Surgery, Department of Surgery, University of Pittsburgh, Pittsburgh, Pennsylvania, USA. ${ }^{6}$ Department of Medicine, Medical College of Wisconsin and Blood Research Institute, Blood Center of Wisconsin, Milwaukee, Wisconsin, USA and ${ }^{7}$ Department of Medicine, Vascular Medicine Institute, University of Pittsburgh, Pittsburgh, Pennsylvania, USA. Correspondence: JS Lee (leejs3@upmc.edu)
} 
such as interleukin (IL)-10, prostaglandin E2 (PGE2), and transforming growth factor beta 1 (TGF- $\beta 1$ ). ${ }^{4,5}$ IL-10 is a key counter-regulatory cytokine that suppresses pro-inflammatory responses during endotoxic shock in mice ${ }^{6}$ and promotes neutrophil apoptosis during severe sepsis in humans. ${ }^{7}$ IL-10 is produced by macrophages upon apoptotic cell contact that is, in part, dependent upon the scavenger receptor CD36. ${ }^{8}$ However, the endogenous factor providing the initial cell-cell contact between macrophage and apoptotic cells necessary for this suppressive cytokine signaling to occur is not clearly defined.

Thrombospondin-1 (TSP-1) is a matricellular, homo-trimeric glycoprotein and a principle constituent of platelet $\alpha$-granules. ${ }^{9}$ TSP- 1 activates latent TGF- $\beta 1^{10}$ but, in an extracellular form, can also serve as a "molecular bridge" by which macrophages, through the scavenger receptor $\mathrm{CD} 36$, in cooperation with the vitronectin receptor (that is, integrin $\alpha \mathrm{v} \beta 3$ ), recognizes apoptotic cells. ${ }^{11}$ Given the potential for numerous molecular interactions, it is not surprising TSP-1 has been ascribed diverse and at times contradictory functions. ${ }^{9}$ Furthermore, the scavenger receptor CD36 recognizes a number of endogenously derived ligands including oxidatively modified lipoproteins, glycated proteins, amyloid-forming peptides, cell-derived microparticles, ${ }^{12}$ in addition to apoptotic cells, ${ }^{13}$ further increasing the complexity of potential biological interactions.

Although normal at birth, mice deficient in TSP $\left(t h b s 1^{-/-}\right)$ were originally reported to develop extensive acute and organizing pneumonia with neutrophil accumulation and hemosiderinladen macrophages in the absence of infection. ${ }^{14}$ It has been suggested that the impaired lung homeostasis in $t h b s 1^{-/-}$mice is due to a failure to activate latent TGF- $\beta 1^{10,14}$ and, therefore, predisposing the mice to impaired tissue repair and unresolved inflammation. However, recent studies have shown that TSP-1 is not required for the activation of TGF- $\beta 1$ in vivo and thbs $1^{-/-}$ mice are not protected from developing either pulmonary fibrosis or myelofibrosis, ${ }^{15,16}$ both disease processes in which TGF- $\beta 1$ has been implicated as a central regulator. Here, we present an alternative mechanism by which thbs $1^{-/-}$mice show impaired lung homeostasis. We show that thbs $1^{-/-}$mice are prone to lung injury due to defective IL-10 production. This impaired IL-10 response is due to a failure in TSP-1 mediated apoptotic cell recognition and occurs despite intact phagocytic mechanisms in vivo. These findings indicate a novel role for TSP-1 triggered IL10 production in lung injury resolution.

\section{RESULTS}

Exaggerated neutrophilic inflammation and defective IL-10 production in thbs $1^{-1-}$ mice following lipopolysaccharide (LPS)-induced lung injury

In contrast to previous report, ${ }^{14}$ we did not observe extensive, spontaneous development of pneumonia in thbs $1^{-/-}$mice. However, thbs $1^{-/-}$mice showed higher total bronchoalveolar lavage (BAL) leukocyte counts ( 1.6 vs. $0.9 \times 10^{5}$ cells per ml), and neutrophil counts $\left(1.1\right.$ vs. $0.1 \times 10^{3}$ cells per ml) under basal conditions (reflected at time 0 in Figures $1 \mathbf{a}$ and $\mathbf{b}$ ), but not BAL total protein (Figure 1c) when compared with wildtype (WT) mice. This difference in phenotype may be related to differences in vivarium conditions from prior report, as our facilities employ high specific pathogen-free conditions. Nevertheless, given the fact that the lungs are continuously exposed to airborne and aspirated noxious particles, our findings lend credence to the general principle set forth by Lawler et al. ${ }^{14}$ that the lack of TSP-1 predisposes the lungs to inflammation. These findings led us to formally examine the possibility that the impaired homeostasis in $t h b s 1^{-/-}$mice ${ }^{14}$ represents prolonged inflammation due to a distinct inability to adequately resolve from injurious stimuli.

The thbs $1^{-1-}$ mice showed increased total leukocyte accumulation within the airspaces on day 1 and day 3 following LPS instillation compared with WT (Figure 1a). Airspace leukocytes were mainly comprised of polymorphonuclear leukocytes neutrophils (Figure 1b), within the airspaces. The defining feature of tissue resolution is the reduction from peak leukocyte infiltration by $\sim 50 \%{ }^{17}$ At day $6, t h b s 1^{-1-}$ mice showed $\sim 13$-fold reduction in BAL neutrophils in contrast to the $\sim 24$-fold reduction observed in WT mice $(2.8$ vs. $0.8 \times 10^{5}$ cells per ml) (Figure $\mathbf{1 b}$ ). Thus, day 6 represents the resolution phase in our model and thbs $1^{-1-}$ mice show impaired ability to fully resolve neutrophilic inflammation. The hallmark of lung injury is barrier dysfunction, and thbs $1^{-1-}$ mice showed increased lung microvascular permeability at days 1 and 3 (Figure 1c) and impaired ability to resolve by day 6 when WT mice have normalized BAL total protein concentrations (Figure 1c). As an independent measure of lung leak, we quantified lung microvascular permeability index utilizing Evan's blue dye extravasation method, and confirmed that thbs $1^{-/-}$mice develop lung injury with prolonged trajectory (Figure 1d) and consistent with histological findings at day 6 following LPS instillation (Figure 1e).

In ARDS, IL-10 is a key anti-inflammatory cytokine found in the airspaces and implicated in limiting the net inflammatory response in human lungs. ${ }^{18-20}$ The airspaces of thbs $1^{-/-}$mice showed blunted IL-10 response during the resolution phase of injury when IL-10 concentrations were increased in WT mice (Figure 1f). Consistent with the relative IL-10 deficiency, proinflammatory cytokines IL-6, KC, and MCP-1 (Figures 1g-i) concentrations were increased at day 6 in thbs $1^{-1-}$ mice although tumor-necrosis factor- $\alpha$, granulocyte colony stimulating factor, and granulocyte-macrophage colony-stimulating factor concentrations were not different (Supplementary Figure 1 online). No differences

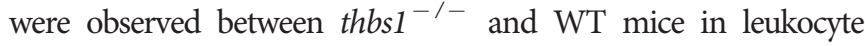
mobilization from the bone marrow following LPS challenge (Supplementary Figure 2A), in vitro neutrophil survival upon serum starvation or following induction by Actinomycin D (Supplementary Figures 2B and C). We also examined gene expression by quantitative real-time PCR in WT and thbs $1^{-1-}$ macrophages in the presence or absence of LPS in vitro. The thbs $1^{-1-}$ macrophages showed exaggerated M1 gene expression (tnf- $\alpha, i l 6, k c$, and $c c l 2)$ compared with WT macrophages. There were minimal changes in M2 gene expression ( $\arg 1$, fizz1, ym1, and il10) from baseline in both WT and thbs $1^{-1-}$ macrophages. Thus, in the absence of TSP-1, there are exuberant $M 1$ gene responses following LPS stimulation (Supplementary Figure 3). 

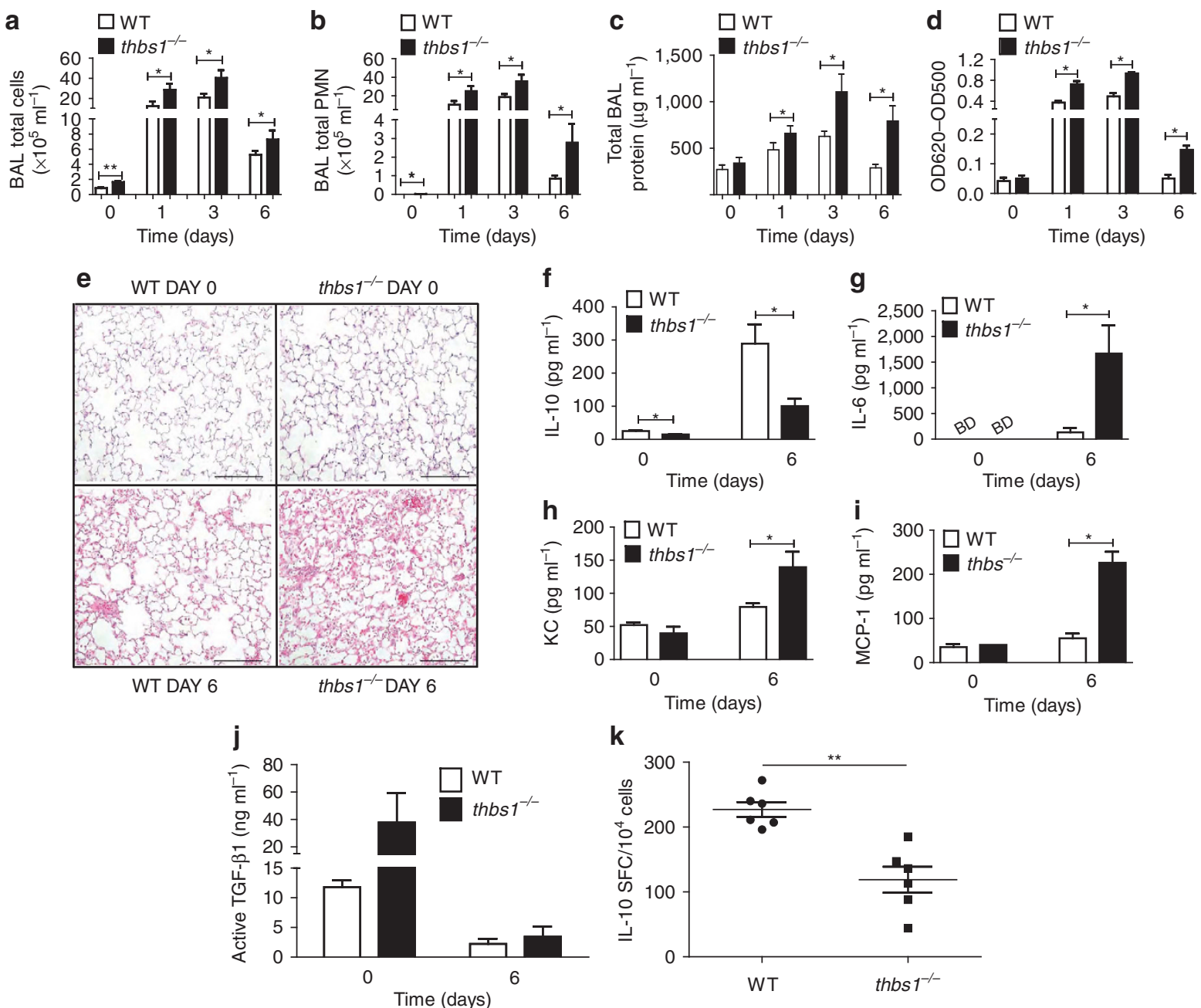

Figure 1 Thrombospondin-1 (TSP-1) is required for effective resolution following lung injury. (a, b) Note that the range of the $y$ axis is separated into two different segments with each lower segment tick interval representing an increase by $1-2 \times 10^{5}$ cells per ml. Each tick interval within the higher segment represents an increase of $20 \times 10^{5}$ cells per ml. (a) Total cell counts per ml, (b) total polymorphonuclear leukocytes (PMN) counts per ml; $n=8-16$ mice per group at day $0,1,3$, and at day 6 . A minimum of two independent experiments was performed for each time points in panels a and $\mathbf{b}$. (c) Total bronchoalveolar lavage (BAL) protein concentrations. $n=4-12$ mice per group at day $0,1,3$, and day 6 . (d) Evan's blue dye extravasation, an indicator of

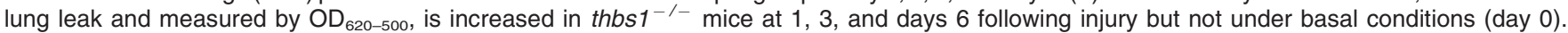
$n=5-7$ mice per group. (e) Representative hematoxylin and eosin (H\&E) of lung tissue sections obtained from wild-type (WT) and mice deficient in thrombospondin-1 (thbs $1^{-/-}$) mice at day 0, 6 post-lipopolysaccharide (LPS) instillation. Scale bar $=100 \mu \mathrm{m}$. (f) IL-10, (g) IL-6, (h) KC and (i) MCP-1 concentrations in BAL. $n=7-8$ mice per group at each time point. Data are represented as mean \pm s.e.m. (j) Bioactive concentrations of soluble transforming growth factor beta 1 (TGF- $\beta 1$ ) as measured by a TGF $\beta 1$-sensitive PAl-1 promoter luciferase reporter assay, $n=3-4$ mice per group performed two independent times. (k) The number of IL-10 producing cells from CD11c-selected BAL cells in WT and thbs $1^{-/-}$mice at day 6 following LPS instillation determined by the enzyme-linked immunosorbent spot assay (ELISPOT). SFC, spot forming cells. $n=4-5$ mice per group. ${ }^{\star \star} P<0.001,{ }^{*} P<0.05$.

As TSP-1 has been previously shown to activate latent TGF$\beta 1,{ }^{10}$ we measured active TGF- $\beta 1$ concentrations in the airspaces but found no differences in thbs1 ${ }^{-1-}$ and WT lungs to account for the impaired resolution (Figure $\mathbf{1 j}$ ). We examined $c d 47^{-1-}$ mice in our model as TSP-1 binds CD47 and TSP-1/CD47 interactions have previously been reported to curtail inflammation. ${ }^{21}$ However, $c d 47^{-/-}$mice did not show enhanced lung inflammation compared with WT mice at the resolution phase of day 6 post-injury (Supplementary Figures $4 \mathrm{~A}-\mathrm{C}$ ). This is consistent with the findings of others that $c d 47^{-1-}$ mice were relatively protected at earlier time points in a lung injury model. ${ }^{22}$ To determine whether alveolar macrophages are involved in IL-10 production at day 6 following injury, we isolated $\mathrm{CD} 11 \mathrm{c}^{+}$cells in the BAL. For the same number of macrophages examined following LPS stimulation in vivo, thbs $1^{-1-}$ macrophages show lower IL-10 responses compared with WT macrophages (Figure 1k). Thus, the findings indicate an inability of thbs $1^{-1-}$ mice to fully curtail neutrophilic inflammation that is associated with a defect in macrophage IL-10 production during the resolution phase of injury.

\section{Reconstitution of IL-10 rescues thbs $1^{-/-}$mice from persistent neutrophilic inflammation and lung leak}

IL-10 is a pleiotropic cytokine and its effects during lung inflammation have been debated. ${ }^{23-25}$ Therefore, we initially tested the responses of $i l 10^{-1-}$ mice to determine whether 
global deficiency of IL-10 results in impaired resolution of inflammation in the LPS-induced lung injury model. Similar to the findings in thbs $1^{-/-}$mice, $i l 10^{-/-}$mice showed prolonged inflammation and increased lung microvascular permeability (Figures 2a-c) during the resolution phase of injury. We next tested whether IL-10 administration could mitigate both neutrophilic inflammation and lung leak in thbs $1^{-/-}$mice following injury. Intraperitoneal administration of recombinant IL-10 reduced total BAL leukocyte counts (Figure 2d), total BAL polymorphonuclear leukocytes counts (Figure 2e) and total BAL protein concentrations (Figure 2f) at day 6 of injury when compared with thbs $1^{-/-}$mice administered vehicle alone (PBS). Reconstitution of IL-10 did not alter cell counts and protein level of WT mice at resolution phase of injury. Thus, correction of the IL-10 defect in thbs $1^{-1-}$ mice restores the ability to adequately resolve inflammation and injury in the lungs near to the levels observed in WT mice.

\section{thbs $1^{-1-}$ alveolar macrophages show defective IL-10 production following intratracheal instillation of apoptotic neutrophils in vivo}

Although macrophages have been shown to produce IL-10 following contact recognition of apoptotic cells ${ }^{8,26}$ as well as following ingestion ${ }^{27}$ in vitro, the role of TSP-1 in mediating this suppressive cytokine signaling in vivo is not known. We tested whether thbs $1^{-1-}$ alveolar macrophages show impairment in either IL-10 production and/or phagocytosis following intratracheal instillation of apoptotic neutrophils generated by serum starvation (Figure 3a). Although thbs $1^{-1-}$ macrophages showed comparable efferocytosis (that is, ingestion of apoptotic cells) to WT mice (Figures $\mathbf{3 b}-\mathbf{d}$ ), thbs $1^{-/-}$alveolar macrophages showed defective IL-10 responses in the airspaces (Figure 3e). These findings indicate that, although TSP-1 is not essential for macrophage engulfment of apoptotic cells, TSP-1 is required for optimal IL-10 production by macrophages upon delivery of apoptotic neutrophils to the lungs.

\section{thbs $1^{-/-}$macrophages show defective IL-10 production following incubation with apoptotic neutrophils in vitro that is neither due to a deficiency in PGE2 or TGF-p1}

To determine whether thbs $1^{-1-}$ macrophages show a defect in the production of other regulatory cytokines following incubation with apoptotic neutrophils, we developed an in vitro co-culture assay. When WT macrophages were incubated in the presence or absence of fresh neutrophils in vitro, IL-10 production was minimal in contrast to incubation in the presence of increasing ratio of apoptotic neutrophils to macrophages (Figure 4a). This effect was accentuated in the presence of LPS-stimulated macrophages (Figure $4 \mathbf{b}$ ). Similar to published report by others, ${ }^{8}$ IL-10 production in WT mice was significantly attenuated in the absence of cell-cell contact between apoptotic neutrophils and macrophages (data not shown). In contrast, thbs $1^{-/-}$ macrophages showed blunted IL-10 production compared with WT macrophages in the presence of increasing numbers of apoptotic neutrophils alone (Figure 4c) or with concomitant stimulation by LPS (Figure 4d). thbs $1^{-1-}$ macrophages did not produce significantly more pro-inflammatory cytokines such as tumor-necrosis factor- $\alpha$, IL-6, or IL-12 compared with WT mice in response to apoptotic polymorphonuclear leukocytes (data not shown). IL-10 production has also
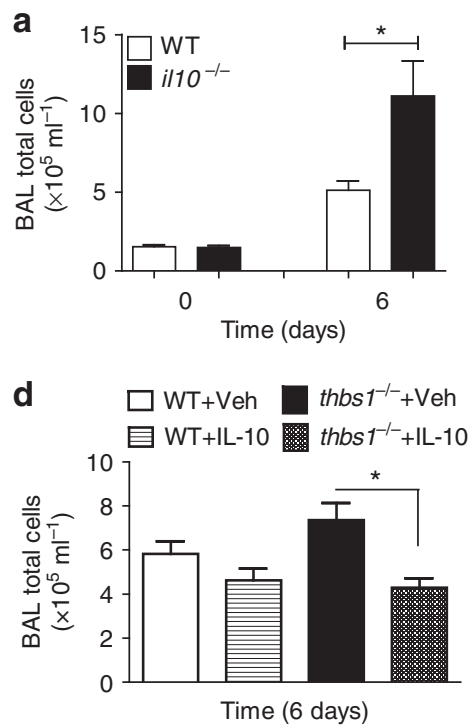
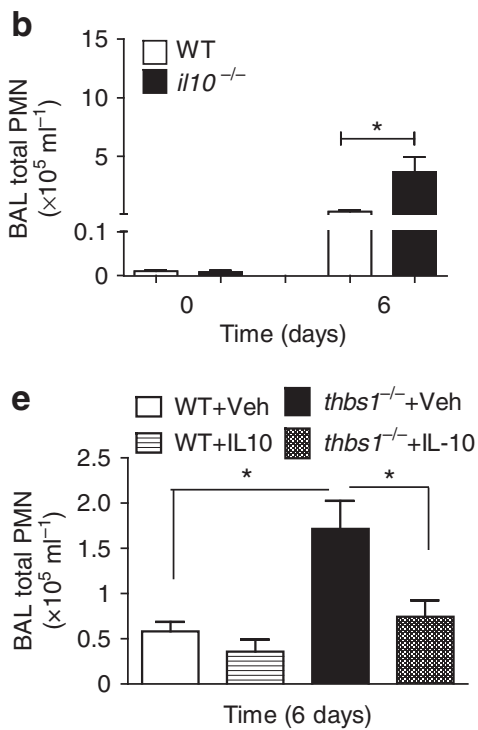
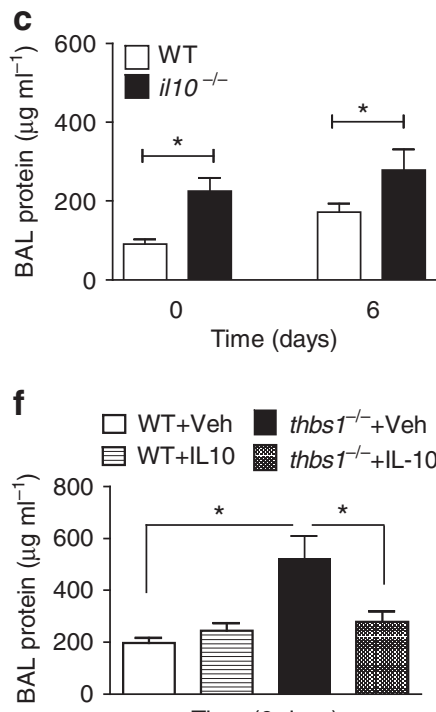

Figure 2 Impaired resolution in $i 10^{-/-}$mice and interleukin (IL)-10 administration attenuates neutrophilic inflammation in the airspaces and lung microvascular permeability of mice deficient in thrombospondin-1 (thbs $1^{-/}$) during the resolution phase of lipopolysaccharide (LPS)-induced lung

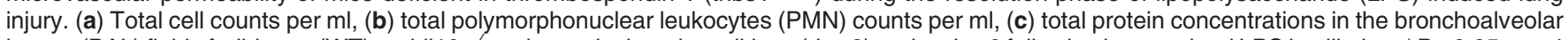
lavage (BAL) fluid of wild-type (WT) and $i 10^{-1}$ mice under basal conditions (day 0 ) and at day 6 following intratracheal LPS instillation. * $P<0.05, n=4$ mice per group at day $0 ; n=7-8$ mice per group at day 6 . (d) Total cell counts per $\mathrm{ml}$, (e) total PMN counts per $\mathrm{ml}$, (f) total protein concentrations in the BAL fluid of WT and thbs $1^{-1-}$ mice at day 6 following intratracheal LPS instillation. WT and thbs $1^{-1-}$ mice were administered either recombinant murine IL$10(1 \mu \mathrm{g})$ or vehicle (phosphate buffer solution; PBS) intraperitoneally at days 0,2 , and 4 following intratracheal LPS. ${ }^{\star} P<0.05, n=7-8$ mice per group at day 6 . Data are represented as mean \pm s.e.m. 

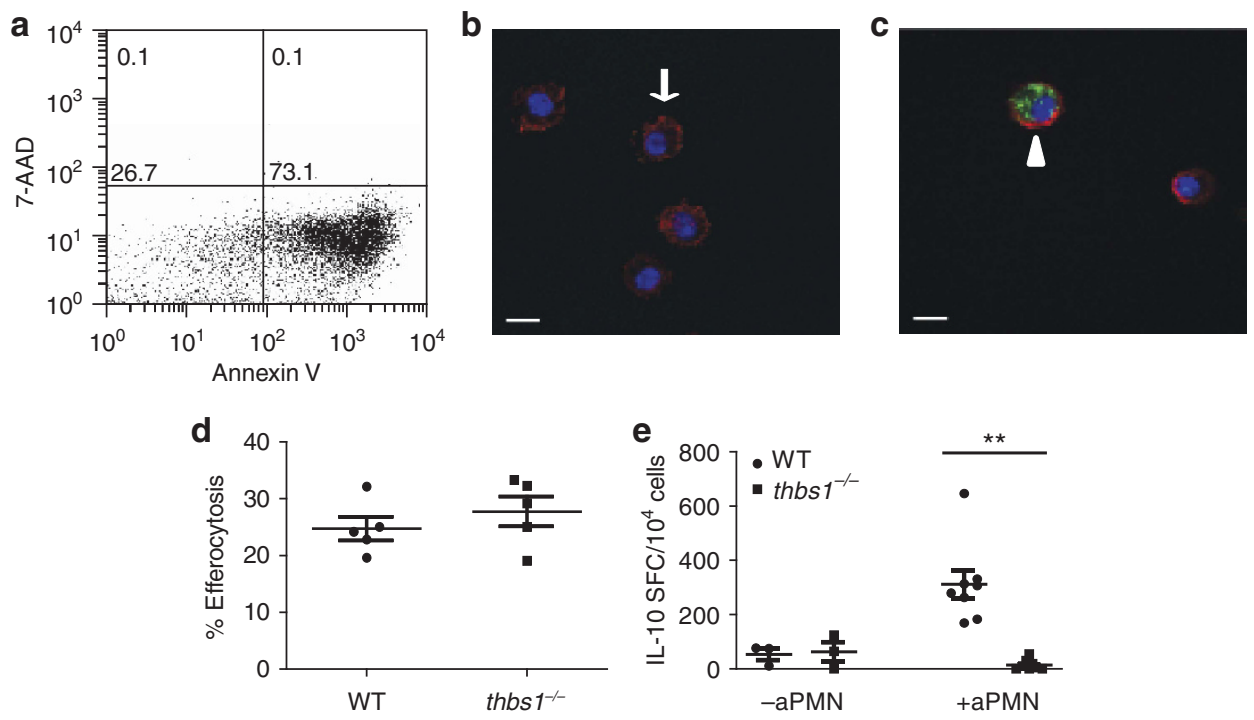

Figure 3 Mice deficient in thrombospondin-1 (thbs $1^{-/-}$) show defective interleukin (IL)-10 production but intact efferocytosis in the lungs following intratracheal instillation of apoptotic neutrophils. (a) \% Of apoptotic neutrophils following $24 \mathrm{~h}$ serum starvation, as indicated by $7-\mathrm{AAD}^{\text {neg }} \mathrm{Annexin}^{+}$ events. (b) Confocal image of alveolar macrophages from naïve wild-type (WT) mice immunostained with CD11c (red), white arrow reflects CD11c ${ }^{+}$ alveolar macrophage. (c) $\mathrm{CD}_{11 \mathrm{c}^{+}}$(red) alveolar macrophages instilled with DDAO-labeled apoptotic neutrophils (green), white arrowhead reflects ingested apoptotic neutrophils. Scale bar $=10 \mu \mathrm{m}$. (d) \% Efferocytosis in WT or thbs $1^{-1-}$ mice. (e) The number of IL-10 producing cells from CD11cenriched bronchoalveolar lavage (BAL) cells in WT and thbs $1^{-1-}$ mice with or without intratracheal instillation of apoptotic neutrophils determined by the enzyme-linked immunosorbent spot assay. SFC, spot forming cells. ${ }^{* \star} P<0.01, n=4-5$ mice per group.

been shown to be mediated by PGE2 following phagocytosis of apoptotic lymphocytes by macrophages. ${ }^{27}$ However, we found similar PGE2 concentrations in WT and thbs $1^{-1-}$ macrophages in the presence (Figure 4e) or absence of LPS (data not shown). In addition, total TGF- $\beta 1$ production was increased

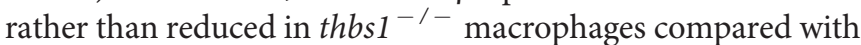
$\mathrm{WT}$ in the presence or absence of apoptotic cells in vitro (Figure 4f). Thus, thbs $1^{-/-}$macrophages show a selective defect in IL-10 production, whereas PGE2 and TGF- $\beta 1$ responses to apoptotic neutrophils show no impairment.

\section{Reconstitution of TSR 2 domain in thbs $1^{-/-}$macrophages restores IL-10 production but inhibition of CD36 signaling prevents full restoration}

TSP-1 contains three contiguous TSP structural homology repeat (TSR) domains. TSR domain 2 contains the structural determinants required for binding to the scavenger receptor CD36. ${ }^{28-30}$ Utilizing purified human TSR domain $2,{ }^{31}$ we show dose-dependent restoration of IL-10 production in LPS stimulated thbs $1^{-/-}$macrophages to WT levels (Figure 5a). CD36 LIMP-II Emp sequence homology (CLESH) domain is a specific region of CD36 that interacts with TSR with high affinity and is a known effective inhibitor of CD36-TSP complex formation and of CD36-dependent cell surface TSP binding. ${ }^{32}$ Pre-treatment with three different recombinant $\mathrm{CD} 36$ fusion proteins containing the CLESH domain attenuated IL-10 production in WT macrophages following incubation with apoptotic neutrophils compared with a control recombinant peptide (Figure 5b). Supporting the finding with the CLESH peptides, we show that knockdown of CD36 expression utilizing small interfering RNA (siRNA) strategy attenuated IL-10 production compared with scramble
RNA (scRNA)-treated macrophages (Figures $5 \mathbf{c}$ and $\mathbf{d}$ ). Collectively, these findings directly establish that TSP-1 interactions with CD36, at least in part, account for IL-10 production by macrophages following encounter with apoptotic neutrophils in vitro.

\section{DISCUSSION}

TSP-1 protein is increased in the airspaces of individuals with ARDS, ${ }^{33}$ but its role in lung injury is not known. Idell et al. ${ }^{33}$ postulated that proteins such as TSP-1 released from stimulated platelets gain entry into the airspaces during lung injury and influence the course of inflammation in patients with ARDS. Still, others have shown that IL-10 is a key anti-inflammatory cytokine found in the airspaces of ARDS patients implicated in curtailing the net inflammatory responses in human lungs following injury from various insults. ${ }^{19}$ Our findings show that TSP-1 aids in the curtailment of inflammatory responses by providing a means by which apoptotic bodies are recognized and trigger macrophage IL-10 production.

thbs $1^{-/-}$alveolar macrophages exhibit an impairment in the ability to produce IL-10 following LPS instillation or direct delivery of apoptotic neutrophils in vivo, and IL-10 administration restores the ability of thbs $1^{-1-}$ mice to resolve both lung inflammation and injury to that of WT mice. Others have shown in vitro that apoptotic cells can induce IL-10 production by monocytes $^{26}$ and macrophages that is, in part, CD36-dependent. ${ }^{8}$ Although TSP-1 is known to serve as a bridging molecule for the recognition of apoptotic cells by macrophages, prior studies examined this role in the context of in vitro phagocytosis ${ }^{11}$ and not IL-10 production. We show that reconstitution of the TSR2 domain of TSP- 1 that recognizes the scavenger receptor CD36 restores IL-10 production in thbs $1^{-/-}$macrophages, and 
a

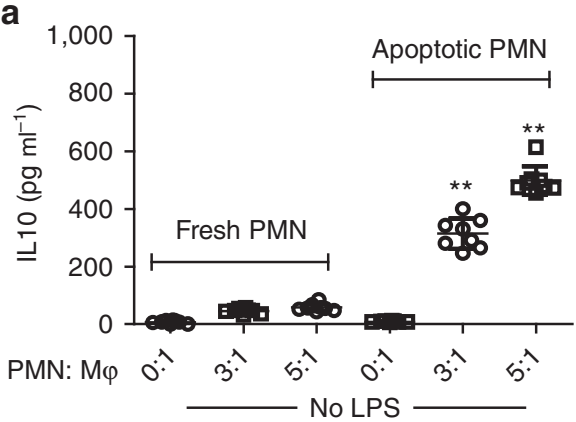

C

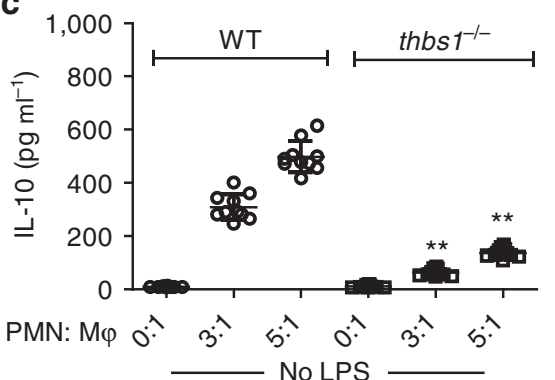

e

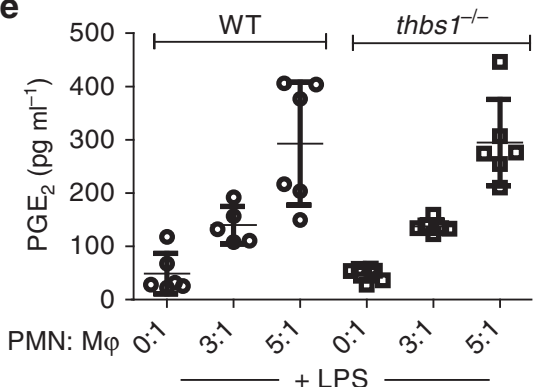

b

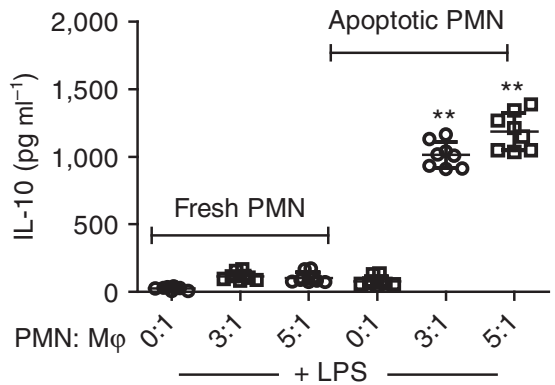

d

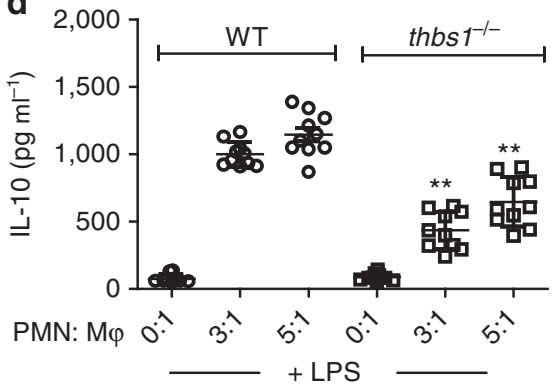

f

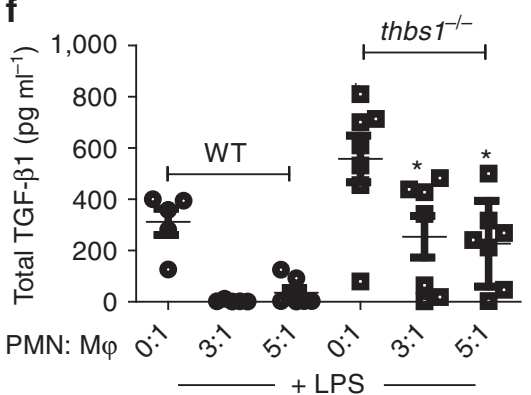

Figure 4 Optimal interleukin (IL)-10 production by macrophages following stimulation with apoptotic neutrophils requires thrombospondin-1 (TSP-1). (a) IL-10 production in $\mathrm{g} \mathrm{ml}^{-1}$, as measured in the supernatant, following incubation of wild-type (WT) macrophages with fresh or apoptotic neutrophils in the absence of lipopolysaccharide (LPS), and (b) following stimulation with LPS. (c) IL-10 production in pg $\mathrm{ml}^{-1}$ following incubation of WT vs. thbs $1^{-/-}$ macrophages with syngeneic apoptotic neutrophils in the absence of LPS, and (d) following stimulation with LPS. (e) Prostaglandin E2 (PGE2) production in $\mathrm{pg} \mathrm{ml}^{-1}$ following incubation of WT vs. thbs $1^{-/-}$macrophages with syngeneic apoptotic neutrophils following stimulation with LPS. (f) Total transforming growth factor beta 1 (TGF- $\beta 1$ ) production in $\mathrm{pg} \mathrm{ml}^{-1}$ following incubation of WT and thbs $1^{-/-}$macrophage with syngeneic apoptotic neutrophils following stimulation of macrophages with LPS before the addition of neutrophils. Data points represent individual wells performed in duplicate, utilizing macrophages combined from 3-4 mice, and neutrophils harvested from 5-6 mice from respective genotype, mean \pm s.e.m. ${ }^{*} P<0.01$, ${ }^{*} P<0.001$, comparison with respective WT control at the same polymorphonuclear leukocytes (PMN):M $\phi$ ratio. Experiment was performed twice with similar results, and the results of one representative experiment are shown.

inhibition of the CD36 CLESH domain that binds TSR prevents full IL-10 responses in WT macrophages. Silencing CD36 expression also blunts IL-10 production in WT macrophages. Our study is the first to directly establish the link between TSP-1 and IL-10 as a distinct mechanism by which apoptotic cells trigger macrophages for injury resolution in vivo.

Although TSP-1 has been shown to be the major activator of TGF- $\beta 1$ in vivo, ${ }^{10}$ the evidence presented here shows that the active form of TGF- $\beta 1$ is detectable in the airspaces of $t h b s 1^{-1-}$ mice. This suggests that other mechanisms are involved in the activation of latent TGF- $\beta 1$ and compensate for the absence of TSP-1 in vivo. In fact, total TGF- $\beta 1$ production is increased in thbs $1^{-1-}$ macrophages compared with WT macrophages under basal conditions in vitro, although the mechanism for this finding remains unclear. Our finding is consistent with recent observations where the absence of TSP-1 does not confer protection in models of fibrosis in which TGF- $\beta 1$ is thought to be causally involved. ${ }^{15,16}$ In both models, increased fibrosis was observed in the absence of TSP- $1 .{ }^{15,16}$

In summary, we show that the cellular recognition of apoptotic cells mediated by TSP- 1 is selective in its ability to trigger IL-10 production. Interestingly, thbs $1^{-/-}$macrophages showed no defect in the engulfment of apoptotic neutrophils in vivo, suggesting that engulfment can occur through other mechanisms that may compensate for the loss of TSP-1. This is not surprising, as multiple pathways for engulfment of apoptotic cells have been identified and efferocytosis is essential for an adequate repair response following injury and the maintenance of homeostasis in tissue. ${ }^{34}$ As lungs fail to resolve optimally following injury when there is a failure of protective 

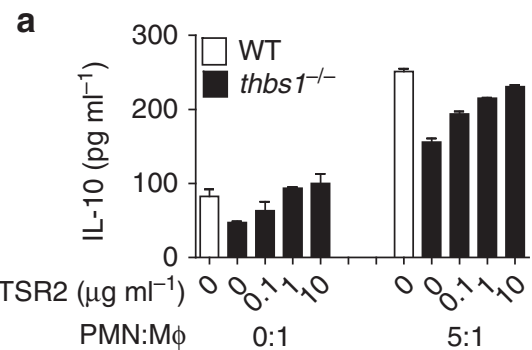

C

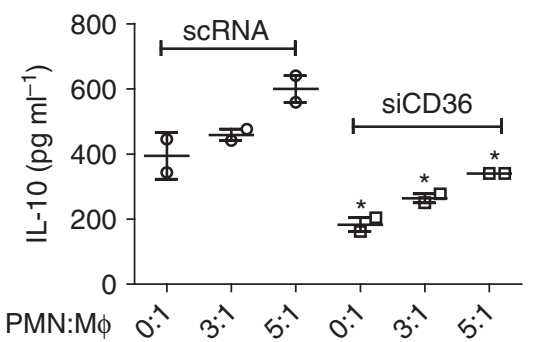

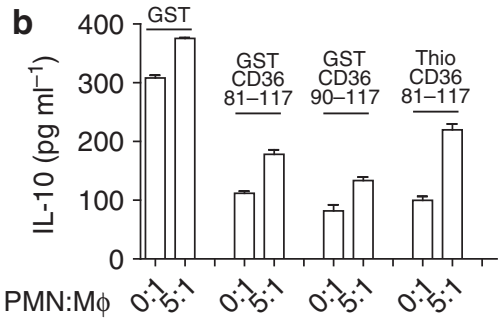

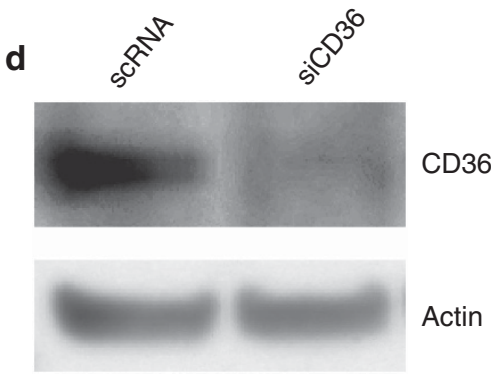

Figure 5 Thrombospondin structural homology repeat 2 (TSR 2) and CD36 engagement is required for macrophage interleukin (IL)-10 production. (a) Macrophages from thbs $1^{-1-}$ mice were pre-incubated with increasing concentrations of TSR domain 2 peptide before lipopolysaccharide (LPS) stimulation and the addition of syngeneic apoptotic neutrophils to wild-type (WT) and thbs $1^{-/-}$macrophages. (b) IL-10 production in pg ml ${ }^{-1}$ following incubation of WT macrophages with glutathione S-transferase (GST) alone, GST-CD36 (81-117), GST-CD36 (90-117), and thioredoxin-CD36 (81-117) peptides containing the CD36 LIMP-II Emp sequence homology (CLESH) domain at $1 \mu \mathrm{g} \mathrm{ml}^{-1}$ each in the presence of LPS before the addition of syngeneic apoptotic neutrophils. (c, d) Macrophages were transfected with small interfering RNA (siRNA) targeted against CD36 and control scramble sequence RNA (scRNA). At $72 \mathrm{~h}$ post-transfection, macrophages were stimulated with LPS $\left(10 \mathrm{ng} \mathrm{ml}^{-1}\right)$ for $1 \mathrm{~h}$ before the addition of neutrophils and IL10 in the supernatant was subsequently detected by enzyme-linked immunosorbent assay (ELISA). Cells lysates were analyzed by SDS-PAGE and immunoblotted with CD36 and $\beta$-actin antibodies. ${ }^{\star} P<0.05$. (a-d) Are representative of two independent experiments. Each condition was performed in duplicates, utilizing macrophages combined from 3-4 mice, and neutrophils harvested from 5-6 mice.

endogenous mechanisms such as TSP-1 triggered IL-10 production, we speculate that TSP-1 provides an additional level of control during inflammation to prevent collateral damage when immature myeloid cells are recruited into tissue and expand in numbers rapidly. It remains to be seen whether persistent lung injury in some individuals represents impaired TSP-1/CD36 interactions.

\section{METHODS}

Animals. C57BL/6J (no. 000664), thbs $1^{-/-}\left(\mathrm{B} 6.129 \mathrm{~S} 2-\mathrm{Th} b s 1^{\text {tmiHyn }} / \mathrm{J}\right.$, no. 006141), and $i l 10^{-1-}$ (B6.129P2-Il10 ${ }^{\text {tml Cgn }} / \mathrm{J}$, no. 00251), $c d 47^{-/-}$ $\left(\mathrm{B} 6.129 \mathrm{~S} 7-C d 47^{t m 1 F p l} / \mathrm{J}\right.$, no. 003173) mice were purchased from The Jackson Laboratory (Bar Harbor, ME). Homozygous breeding colonies for thbs $1^{-/-}$mice were established subsequently at the University of Pittsburgh. All experimental procedures were performed in age (8-12 week old) and gender-matched mice. The animals were housed and maintained in a pathogen-free environment and studies were conducted in accordance with the Institutional Animal Care and Use Committee at the University of Pittsburgh.

LPS-induced lung inflammation and injury model. Lipopolysacchride (LPS; from Escherichia coli 011:B4; List Biological Laboratories, Campbell, CA) at a dose of $3 \mathrm{mg} \mathrm{kg}^{-1}$ was dissolved in nuclease free water and instilled into the lungs by direct visualization using a sterile $200 \mu \mathrm{l}$ pipet with filtered tip positioned just above the vocal cords. Method for the delivery of LPS intratracheally has been previously described. ${ }^{35}$ In some experiments, $1 \mu \mathrm{g}$ of recombinant mouse IL10 (eBioscience, San Diego, CA) or PBS vehicle was intraperitoneally injected to WT and thbs $1^{-1-}$ mice at day 0,2 , and 4 following LPS instillation.

Mouse necropsies and bronchoalveloar lavage. Animals were euthanized at specified time points with isoflurane inhaled anesthetic overdose within a closed container system or $100 \mathrm{mg} \mathrm{kg}^{-1}$ pentobarbital administered intraperitoneally followed immediately by exsanguination via intracardiac puncture. Mouse necropsy, lung tissue processing, and BAL have been previously described. ${ }^{36}$ For histology, the right lung was stained with Hematoxylin 2 and Eosin Y (RichardAllan Scientific, Kalamazo, MI). BAL total protein concentration was determined using the Pierce BCA Protein Assay Kit (Thermo Scientific, Rockford, IL).

Evans blue extravasation in the lungs. Pulmonary microvascular permeability index was measured using a modification of the Evans blue dye extravasation technique. ${ }^{37}$ Briefly, WT and thbs $1^{-/-}$mice received $100 \mu \mathrm{l}$ of a $1 \%$ solution of Evans blue dye (Sigma-Aldrich, St Louis, MO) through the lateral tail vein 30 min before euthanization. The pulmonary circulation was perfused with $5 \mathrm{ml}$ ice cold $0.9 \%$ NS via the right ventricle. Lungs were removed and extracted in $1 \mathrm{ml}$ of formamide at $55^{\circ} \mathrm{C}$ overnight. Evans blue content was determined by calculating the OD620 minus OD500 as previously published. ${ }^{37}$

Cytokine determinations. BAL cytokines were assayed using the Bioplex cytokine assay platform (Bio-Rad, Hercules, CA) and select cytokines were subsequently confirmed by ELISA (IL-10, IL-6, KC, MCP-1, tumor-necrosis factor- $\alpha$, granulocyte colony stimulating factor, and granulocyte-macrophage colony-stimulating factor) using DuoSet kits (R\&D Systems, Minneapolis, MN). For the in vitro coculture assays, IL-10 and total TGF- $\beta 1$ concentrations were measured by ELISA using DuoSet kits (R\&D Systems) and PGE2 was measured by an EIA assay from Cayman Chemical (Ann Arbor, MI).

TGF- $\beta 1$ bioactivity assay of BAL samples. TGF- $\beta 1$ activity was determined using a plasminogen activator inhibitor-1 (PAI-1) promoter luciferase reporter assay as previously described ${ }^{38}$ and a kind gift from Dr Daniel Rifkin.

In vitro apoptotic neutrophil-macrophage assay. Neutrophils and macrophages were harvested from the peritoneum of mice 24 and $96 \mathrm{~h}$ 
following intra-peritoneal injection with $3 \mathrm{ml}$ thioglycollate. Cell counts were performed manually using a hemacytometer. Cytospins confirmed $>90 \%$ neutrophils at $24 \mathrm{~h}$ harvest, and $>95 \%$ macrophages at the $96 \mathrm{~h}$ harvest. Apoptosis was induced by serum starvation for $24 \mathrm{~h}$ or, in select experiments, with actinomycin (Sigma-Aldrich) $10 \mathrm{~nm}$ for $5 \mathrm{~h} .{ }^{39}$ Macrophages were plated onto 48 -well plates in RPMI 1640 (BioWhittaker, Walkersville, MD) with $10 \%$ FBS or AIM-V serum-free media (Invitrogen, Grand Island, NY) and incubated for $18 \mathrm{~h}$. Media was removed and apoptotic neutrophils were added into culture at the prescribed ratio. In select experiments, macrophages were pre-stimulated with LPS for $1 \mathrm{~h}\left(011: \mathrm{B} 4,10 \mathrm{ng} \mathrm{ml}^{-1}\right)$ before the addition of neutrophils. After $18 \mathrm{~h}$, supernatants from the co-culture assay were collected for ELISA. In other experiments, cells were preincubated with different concentrations of TSR2 domain peptide, GST-CLESH, or Thio-CLESH before LPS stimulation and addition of neutrophils.

In vivo phagocytosis assay. Blood was obtained from healthy volunteers following informed consent and this study was approved by the University of Pittsburgh Institutional Review Board. Human neutrophils from fresh blood were separated by gradient centrifugation using Ficoll-Paque Plus (GE Healthcare Life Sciences, Pittsburgh, PA). RBC was lysed with $1 \times$ RBClysis buffer twice. Purity of neutrophils was $>90 \%$ by cytospin. Neutrophils were cultured in RPMI 1640 without serum in $5 \% \mathrm{CO}_{2}$ at $37^{\circ} \mathrm{C}$ overnight. Apoptotic neutrophils were assayed by flow cytometry and $>90 \%$ by Annexin V and 7-ADD staining. Neutrophils were labeled with $0.5 \mu \mathrm{M}$ DDAO SE (Invitrogen Molecular Probes) according to prior published methods ${ }^{40}$ and were intratracheally instilled to mice (6 million cells per mouse). BAL cells were harvested the following day and immuno-stained with anti-mouse CD11c FITC (BD Biosciences, San Jose, CA), and data acquired on LSRII Fortessa (BD Biosciences). Slides for confocal microscopy were prepared by cytospin, and fixed with $2 \%$ paraformaldehyde for $10 \mathrm{~min}$. Slides were immuno-stained with hamster anti-mouse $\mathrm{CD} 11 \mathrm{c}$ antibody. Images were taken under confocal microscope and \% efferocytosis calculated by manual counting of double positive cells over total CD11c immunostained cells.

Mouse IL-10 ELISPOT assay. Neutrophils from the peritoneum were obtained as described above. After inducing apoptosis, cells were intratracheally instilled to WT and thbs $1^{-1-}$ mice ( 3 million cells per mouse). Twenty-four hours later, BAL samples were harvested and cells were selected by CD11c magnetic-bead purification (Miltenyi Biotec Automacs Technology, Auburn, CA). In some experiments, BAL cells were selected by CD11c purification at day 6 following LPS instillation. The purity of alveolar macrophages following CD $11 \mathrm{c}$ selection was quantified at $95.9 \pm 2.0 \%(n=4$ examinations) by cytospin examination. After incubation for $48 \mathrm{~h}$, mouse IL-10 was detected by ELISPOT according to manufacturers' instruction. Splenocytes stimulated with LPS $\left(1 \mu \mathrm{g} \mathrm{ml}^{-1}\right)$ in vitro were used as positive controls.

siCD36 transfection. Macrophages were transfected with siRNA against CD36 (50 nM) and control scRNA, using HiPerFect (Qiagen, Valencia, CA) according to the manufacturer's instructions. The transfection efficiency was determined by protein expression by immunoblotting with the corresponding antibodies $72 \mathrm{~h}$ later. Macrophage and apoptotic neutrophils co-culture assays were performed using scRNA as controls. siCD36 and scRNA were purchased from Dharmacon (Thermo Scientific, Rockford, IL) and these siRNAs are pools of four siRNA duplexes (smartpools).

Western blot analysis. Rabbit anti CD36 (Novus Biologicals, Littleton, CO) was used for immunoblotting. Proteins were resolved by SDS-PAGE under reducing conditions, transferred to a nitrocellulose membrane, and blocked with 5\% nonfat dried milk in TBS containing $0.05 \%$ Tween 20 for $1 \mathrm{~h}$. Immunoblotting was performed by incubating membranes in blocking solution containing primary antibodies at $4{ }^{\circ} \mathrm{C}$ overnight. Following extensive washing with TBS containing $0.05 \%$ Tween 20, the membranes were incubated with horseradish peroxidase-conjugated secondary antibodies (Cell Signaling Technology, Boston, MA) for $1 \mathrm{~h}$ at room temperature. An enhanced chemiluminescence detection kit (Thermo Scientific) was used to visualize bound antibody.

Adoptive transfer studies. B6.SJL-Ptprca Pep3b/BoyJ recipients expressing the CD45.1 allele were irradiated with 1000 cGy in a Cesium irradiator. One day following irradiation, a 50:50 mixture of bone marrow cells harvested from the femur of euthanized thbs $1^{-1-}$ (expressing the CD45.2 allele) and WT (CD45.1 allele; B6.SJL-Ptprca $P e p 3 b /$ BoyJ ) mice were injected at $25 \times 10^{6}$ per $200 \mu \mathrm{l}$ by tail vein into B6.SJL-Ptprca Pep3b/BoyJ recipients. At day 45, B6.SJL-Ptprca Pep3b/ BoyJ recipients were intracheally instilled with $3 \mathrm{mg} \mathrm{kg}^{-1} \mathrm{LPS}$. At days 3 and 6 following injury, the \% of CD45.2 and CD45.1 cells were assayed both in the peripheral blood and BAL utilizing PE anti-mouse CD45.1 and FITC anti-mouse CD45.2 (BD Pharmingen, San Diego, CA).

Statistics. Results are reported as the mean plus or minus s.e.m. unless otherwise indicated. A two-tailed Student's $t$-test was used to determine significance using GraphPad Prism software version 5.0 (La Jolla, CA). A $P$-value less than 0.05 was considered significant.

SUPPLEMENTARY MATERIAL is linked to the online version of the paper at http://www.nature.com/mi

\section{ACKNOWLEDGEMENTS}

Funding sources include NIH DK072506 (JMP), GM082852 (MRR), HL086884 (JSL), HL086884-03S1 (JSL), Flight Attendant Medical Research Institute (JSL), Pilot Project Program in Hemostasis and Vascular Biology through UL1 RR024153 and UL1 TR000005 (University of Pittsburgh CTSI), the Vascular Medicine Institute, the Hemophilia Center of Western Pennsylvania, and the Institute for Transfusion Medicine.

\section{DISCLOSURE}

The authors declared no conflict of interest.

(c) 2014 Society for Mucosal Immunology

\section{REFERENCES}

1. Ranieri, V.M. et al. Acute respiratory distress syndrome: the Berlin Definition. JAMA 307, 2526-2533 (2012).

2. Fowler, A.A. et al. Adult respiratory distress syndrome: risk with common predispositions. Ann. Intern. Med. 98, 593-597 (1983).

3. Martin, T.R., Nakamura, M. \& Matute-Bello, G. The role of apoptosis in acute lung injury. Crit. Care Med. 31, S184-S188 (2003).

4. Fadok, V.A. et al. Macrophages that have ingested apoptotic cells in vitro inhibit proinflammatory cytokine production through autocrine/paracrine mechanisms involving TGF-beta, PGE2, and PAF. J. Clin. Invest. 101, 890-898 (1998).

5. Savill, J., Dransfield, I., Gregory, C. \& Haslett, C. A blast from the past: clearance of apoptotic cells regulates immune responses. Nat. Rev. Immunol. 2, 965-975 (2002).

6. Berg, D.J. et al. Interleukin-10 is a central regulator of the response to LPS in murine models of endotoxic shock and the Shwartzman reaction but not endotoxin tolerance. J. Clin. Invest. 96, 2339-2347 (1995).

7. Keel, M. et al. Interleukin-10 counterregulates proinflammatory cytokineinduced inhibition of neutrophil apoptosis during severe sepsis. Blood $\mathbf{9 0}$, 3356-3363 (1997).

8. Chung, E.Y. et al. Interleukin-10 expression in macrophages during phagocytosis of apoptotic cells is mediated by homeodomain proteins Pbx1 and Prep-1. Immunity 27, 952-964 (2007).

9. Bornstein, P. Diversity of function is inherent in matricellular proteins: an appraisal of thrombospondin 1. J. Cell Biol. 130, 503-506 (1995).

10. Crawford, S.E. et al. Thrombospondin-1 is a major activator of TGF-beta1 in vivo. Cell 93, 1159-1170 (1998). 
11. Savill, J., Hogg, N., Ren, Y. \& Haslett, C. Thrombospondin cooperates with CD36 and the vitronectin receptor in macrophage recognition of neutrophils undergoing apoptosis. J. Clin. Invest. 90, 1513-1522 (1992).

12. Ghosh, A. et al. Platelet CD36 surface expression levels affect functional responses to oxidized LDL and are associated with inheritance of specific genetic polymorphisms. Blood 117, 6355-6366 (2011).

13. Silverstein, R.L. \& Febbraio, M. CD36, a scavenger receptor involved in immunity, metabolism, angiogenesis, and behavior. Sci. Signal. 2, re3 (2009).

14. Lawler, J. et al. Thrombospondin-1 is required for normal murine pulmonary homeostasis and its absence causes pneumonia. J. Clin. Invest. 101, 982-992 (1998).

15. Ezzie, M.E. et al. Thrombospondin-1-deficient mice are not protected from bleomycin-induced pulmonary fibrosis. Am. J. Respir. Cell Mol. Biol. 44, 556-561 (2011).

16. Evrard, S. et al. Thrombospondin-1 is not the major activator of TGF-beta1 in thrombopoietin-induced myelofibrosis. Blood 117, 246-249 (2011).

17. Schwab, J.M., Chiang, N., Arita, M. \& Serhan, C.N. Resolvin E1 and protectin D1 activate inflammation-resolution programmes. Nature 447 , 869-874 (2007).

18. Armstrong, L. \& Millar, A.B. Relative production of tumour necrosis factor alpha and interleukin 10 in adult respiratory distress syndrome. Thorax $\mathbf{5 2}$, 442-446 (1997).

19. Donnelly, S.C. et al. The association between mortality rates and decreased concentrations of interleukin-10 and interleukin-1 receptor antagonist in the lung fluids of patients with the adult respiratory distress syndrome. Ann. Intern. Med. 125, 191-196 (1996).

20. Park, W.Y. et al. Cytokine balance in the lungs of patients with acute respiratory distress syndrome. Am. J. Respir. Crit. Care Med. 164 1896-1903 (2001).

21. Doyen, $\mathrm{V}$. et al. Thrombospondin 1 is an autocrine negative regulator of human dendritic cell activation. J. Exp. Med. 198, 1277-1283 (2003).

22. Su, X., Johansen, M., Looney, M.R., Brown, E.J. \& Matthay, M.A. CD47 deficiency protects mice from lipopolysaccharide-induced acute lung injury and Escherichia coli pneumonia. J. Immunol. 180, 6947-6953 (2008).

23. Greenberger, M.J. et al. Neutralization of IL-10 increases survival in a murine model of Klebsiella pneumonia. J. Immunol. 155, 722-729 (1995).

24. Sawa, T. et al. IL-10 improves lung injury and survival in Pseudomonas aeruginosa pneumonia. J. Immunol. 159, 2858-2866 (1997).

25. Chmiel, J.F. et al. IL-10 attenuates excessive inflammation in chronic Pseudomonas infection in mice. Am. J. Respir. Crit. Care Med. 160, 2040-2047 (1999).
26. Voll, R.E. et al. Immunosuppressive effects of apoptotic cells. Nature $\mathbf{3 9 0 ,}$ 350-351 (1997).

27. Medeiros, A.I., Serezani, C.H., Lee, S.P. \& Peters-Golden, M. Efferocytosis impairs pulmonary macrophage and lung antibacterial function via PGE2/ EP2 signaling. J. Exp. Med. 206, 61-68 (2009).

28. Tolsma, S.S. et al. Peptides derived from two separate domains of the matrix protein thrombospondin-1 have anti-angiogenic activity. J. Cell Biol. 122, 497-511 (1993).

29. Dawson, D.W. et al. CD36 mediates the In vitro inhibitory effects of thrombospondin-1 on endothelial cells. J. Cell Biol. 138, 707-717 (1997),

30. Asch, A.S., Silbiger, S., Heimer, E. \& Nachman, R.L. Thrombospondin sequence motif (CSVTCG) is responsible for CD36 binding. Biochem. Biophys. Res. Commun. 182, 1208-1217 (1992).

31. Klenotic, P.A., Page, R.C., Misra, S. \& Silverstein, R.L. Expression, purification and structural characterization of functionally replete thrombospondin-1 type 1 repeats in a bacterial expression system. Protein Exp. Purification 80, 253-259 (2011).

32. Frieda, S., Pearce, A., Wu, J. \& Silverstein, R.L. Recombinant GST/CD36 fusion proteins define a thrombospondin binding domain. Evidence for a single calcium-dependent binding site on CD36. J. Biol. Chem. 270, 2981-2986 (1995)

33. Idell, S. et al. Platelet-specific alpha-granule proteins and thrombospondin in bronchoalveolar lavage in the adult respiratory distress syndrome. Chest 96, 1125-1132 (1989).

34. Ravichandran, K.S. \& Lorenz, U. Engulfment of apoptotic cells: signals for a good meal. Nat. Rev. Immunol. 7, 964-974 (2007).

35. Lee, J.S. et al. The Duffy antigen modifies systemic and local tissue chemokine responses following lipopolysaccharide stimulation. J. Immunol. 177, 8086-8094 (2006).

36. Mangalmurti, N.S. et al. Loss of red cell chemokine scavenging promotes transfusion-related lung inflammation. Blood 113, 1158-1166 (2009).

37. Camerer, E. et al. Sphingosine-1-phosphate in the plasma compartment regulates basal and inflammation-induced vascular leak in mice. J. Clin. Invest. 119, 1871-1879 (2009).

38. Abe, M. et al. An assay for transforming growth factor-beta using cells transfected with a plasminogen activator inhibitor-1 promoter-luciferase construct. Anal. Biochem. 216, 276-284 (1994).

39. Martin, S.J. et al. Early redistribution of plasma membrane phosphatidylserine is a general feature of apoptosis regardless of the initiating stimulus: inhibition by overexpression of Bcl-2 and Abl. J. Exp. Med. 182, 1545-1556 (1995).

40. Poe, S.L. et al. STAT1-regulated lung MDSC-like cells produce IL-10 and efferocytose apoptotic neutrophils with relevance in resolution of bacterial pneumonia. Mucosal Immunol. 6, 189-199 (2013). 